\title{
Influence of host galaxy morphology on the properties of Type la supernovae from JLA and Pantheon compilations
}

\author{
Maria Pruzhinskaya* \\ Lomonosov Moscow State University, Sternberg Astronomical Institute, Universitetsky pr. 13, \\ Moscow 119234, Russia \\ E-mail: pruzhinskaya@gmail.com
}

\author{
Alexandra Novinskaya \\ Lomonosov Moscow State University, Faculty of Physics, Leninskie Gory, 1-2, Moscow, 119991, \\ Russia \\ E-mail: alexandranovinskaya@gmail.com
}

\section{Philippe Rosnet}

Université Clermont Auvergne, CNRS/IN2P3, LPC, Clermont-Ferrand, France

E-mail: Philippe.ROSNETCclermont.in2p3.fr

\section{Nicoleta Pauna}

Université Clermont Auvergne, CNRS/IN2P3, LPC, Clermont-Ferrand, France

E-mail: nicoleta.pauna@clermont.in2p3.fr

In this work we show the correlation between host galaxy morphology and light curve parameters of Type Ia supernovae. The analysis is based on two most recent cosmological samples of supernovae: JLA (Joint Light-curve Analysis) and Pantheon. We confirm that the stretch-parameter depends on the host morphology, but there is no any correlation for the colour. This result is in the line of other analyses showing the environment dependence of Type Ia supernova light curves. We stress the importance of including the host morphology term to the supernova standardization procedure.

Multifrequency Behaviour of High Energy Cosmic Sources - XIII - MULTIF2019

3-8 June 2019

Palermo, Italy

${ }^{*}$ Speaker. 


\section{Introduction}

Among the other types of supernovae, Type Ia supernovae (SNe Ia) have less luminosity dispersion at maximum light and show higher optical luminosities. These two properties allowed to use them as cosmological distance indicators that led to the discovery of the accelerating expansion of the Universe [1,2]. The most recent analysis of SNe Ia indicates that considering a flat $\Lambda \mathrm{CDM}$ cosmology, our Universe is accelerating with $\Omega_{\Lambda}=0.702 \pm 0.022$ [3].

In the early studies it was widely assumed that $\mathrm{SNe}$ Ia have universal light curves and a constant absolute magnitude at maximum ("standard candles"). This was explained by the similarity of the physical processes that lead to the outburst phenomenon. Generally, the outburst is a thermonuclear explosion of a $\mathrm{C}-\mathrm{O}$ white dwarf whose mass has become close to or larger than the Chandrasekhar mass. In fact, after the detailed observations of a large number of supernovae had been accomplished, it became clear that the absolute magnitude at maximum can vary within $\sim 1$ mag. The reasons of luminosity dispersion could be different. First, the mechanism of mass increase by white dwarf is not universal: it can be due to the matter accretion on the white dwarf from the companion star (so-called "single-degenerate (SD) scenario") or the merger of two white dwarfs ("double-degenerate (DD) scenario"). Additionally there exist some alternative scenarios, like sub-Chandrasekhar, that is usually associated with weak explosions. These scenarios have internal freedom that results in significant variations in observed light curves of SNe Ia: like point of deflagration-to-detonation transition (for SD scenario), or difference in total mass (for DD scenario). Another important factor which could violate the "standard candle" hypothesis is dust. Dust around the supernovae, as well as in the host galaxy, surely affects light curve behavior. The distribution and properties of dust in host galaxies of supernovae could be different from that in the Milky Way. In addition, the initial chemical composition of the progenitor stars also complicates the picture. A lower metallicity involves an increase of the Chandrasekhar limit and could affect the explosion process. For example, in elliptical galaxies or in halo of spiral galaxies only old, i.e. metal-poor, stars with an age comparable to that of the Universe are located; on the contrary in the star formation regions of spiral galaxies there are young metal-rich stars. The above-listed factors (age, chemical composition of the region around SN, presence of dust) could be considered as environmental effects.

Fortunately, it was established that supernovae are "standardized candles" (see Section 2), that allowed to improve a lot the accuracy of distance measurements. However, the dispersion on the Hubble diagram still remains. For instance, in the most recent cosmological analysis all $\mathrm{SNe}$ are standardized with an intrinsic residual dispersion of about $0.11 \mathrm{mag}$. It could be due to the environmental effects that are not accounted by current standardization methods. Therefore, the SN Ia standardization procedure is one of the main sources of systematic uncertainties in the cosmological results.

\section{Rust-Pskovskii relation}

The discovery of the accelerating expansion of the Universe would not have been possible without the discovery of one of the most important relation in supernova cosmology - the relation between the peak luminosity of Type Ia supernovae and their luminosity decline rate after the 
maximum light. The history of this relation is quite long and interesting (see [4]). The relation was independently discovered by the American statistician and astronomer Bert Woodard Rust (Fig. 1a) and the Soviet astronomer Yury Pavlovich Pskovskii (Fig. 1b) in the 1970th [5, 6, 7]. Using a limited sample of Type I supernovae they were able to show that the brighter the supernova, the slower its luminosity declines after the maximum (stretch correction). Only with the appearance of CCD astronomy could Mark Phillips re-inspect this relation on the new level of accuracy using the better sample of supernovae [8]. His investigations confirmed the idea earlier proposed by Rust and Pskovskii.

Later it was shown that SN Ia absolute brightness depends on the colour as well (colour correction; [9, 10]). To take into account stretch and colour corrections and, therefore, to determine the absolute magnitude of each SN Ia at maximum, different standardization methods have been developed. We use SALT2 [11] parameters $x_{1}$ describing the time stretching of the light-curve and the colour offset with respect to the average at the date of maximum luminosity in $B$-band, $c=(B-V)_{\max }-\langle B-V\rangle$. We adopt the classical standardization of the distance modulus $(\mu)$ :

$$
\mu=m_{B}^{*}-M_{B}+\alpha x_{1}-\beta c
$$

where $m_{B}^{*}$ - value of the $B$-band apparent magnitude at maximum light, $x_{1}$ - stretch-parameter, $c$ - colour parameter. $M_{B}$ is a standardized absolute magnitude of the $\mathrm{SNe}$ Ia in $B$-band for $x_{1}=c=0, \alpha$ and $\beta$ describe, consequently, the stretch and colour law for the whole sample.

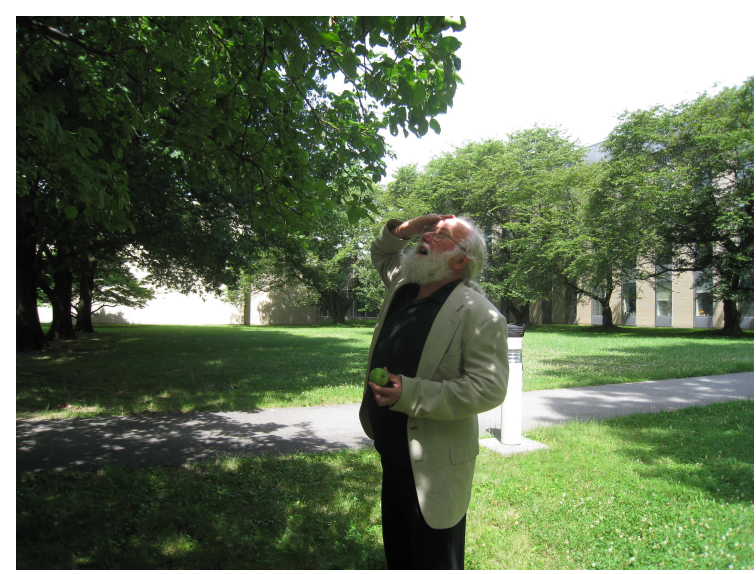

(a)

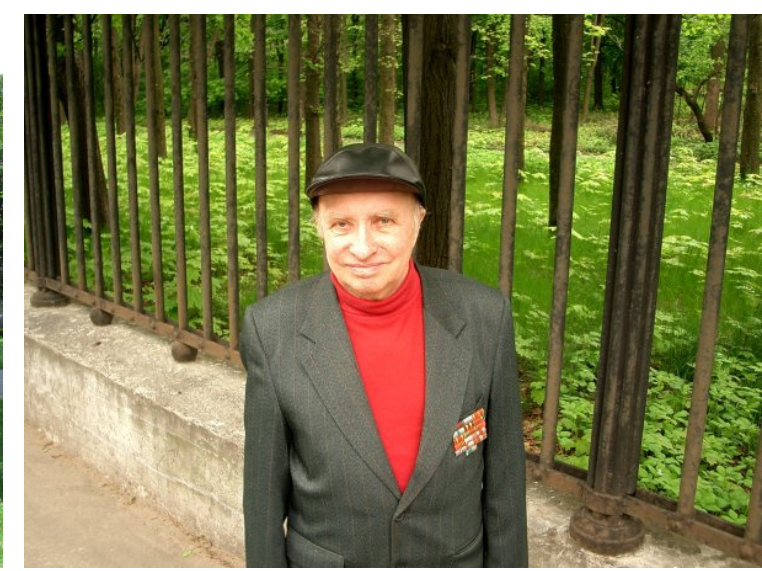

(b)

Figure 1: (a) Bert Woodard Rust. The photo is provided by prof. Rust from his personal archive; (b) Yury Pavlovich Pskovskii $(01.02 .1926$ - 21.07.2004). The photo is taken from http://www.astronet.ru/db/msg/1211317.

\section{Environmental effects}

\subsection{Local vs. global parameters}

The environment of SNe Ia can be characterise by different values that we roughly divide as local and global parameters. The local parameters are local colour, local star formation rate (SFR), local specific SFR, etc. On the other side, characteristics as host galaxy morphology, stellar mass, 
SFR, and galactocentric distance can be referred as global parameters. It is obvious that local parameters provide the more accurate description of the SN environment. However, the current state of the data processing and the resolution of the largest telescopes do not allow measuring local parameters at medium and high redshifts with a good accuracy. That is why study of influence of the local parameters on the SNe Ia properties are based on low-redshift supernova samples. For example, the most recent analysis of the local specific SFR in $1 \mathrm{kpc}$ region around supernovae is done for 141 objects of the Nearby Supernova Factory [12] with redshift $0.02<z<0.08$ [13]. From that point it is more expedient to use global parameters, for example, host galaxy morphology. At the moment, it is possible to determine the morphology of the most distant Hubble galaxies with $z>1$ [14], which makes the study of host morphology impact possible even for cosmological supernovae.

Moreover, the number of discovered supernovae increases dramatically. In epoch of LSST millions of SNe will be discovered every year. In this sense the accurate measurements of the local environmental parameters for each supernovae become very expensive since it requires time on the largest telescopes. The global parameters on the contrary are easier to obtain, for example, processing the images of wide-field photometric surveys with use of traditional astronomical methods as well as machine learning techniques (e.g., [15]).

It is worth to stress that local and global parameters correlate to each other. The comparison between host morphological type and local SFR in $1 \mathrm{kpc}$ region around supernovae for nearby supernova sample [16], is shown in Fig. 2. To plot Fig. 2 we determined the supernova host morphology using SIMBAD ${ }^{1}$, HyperLEDA $^{2}$, and $\mathrm{NED}^{3}$ astronomical databases. See also figure 10 of [17] for the dependence between the local $U-V$ rest-frame colour in a region of $3 \mathrm{kpc}$ around the supernova and the stellar mass of the host galaxy of SNLS (SuperNovae Legacy Survey), SDSS (Sloan Digital Sky Survey), and low-redsfiht supernovae.

Taking into account all of the above, in this work we use host galaxy morphology to describe the supernova environment. We study its impact on two cosmological samples of supernovae: Joint Light-curve Analysis (JLA; [18]) and Pantheon [3].

\subsection{JLA supernova sample}

JLA sample of supernovae includes 740 spectroscopically confirmed SNe Ia up to the redshift $z \simeq 1.3$. Supernovae are collected from nearby, SDSS, SNLS, and HST (Hubble Space Telescope) surveys [18]. According to this analysis the Universe is accelerating with $\Omega_{\Lambda}=0.705 \pm 0.034$ for $\Lambda$ CDM cosmology.

In [19] the host galaxy morphology for $192 \mathrm{SNe}$ Ia from the JLA sample was derived. The supernovae were divided into three categories depending on host morphology: elliptical/lenticular (E/L), early-type spiral (ES), and late-type spiral galaxies (LS).

It was found that:

- Stretch parameter $x_{1}$ is closely correlated with host morphology: the low-stretch (fast decline) SNe usually exploded in E/L galaxies, i.e. in old stellar population environment.

\footnotetext{
${ }^{1}$ http://simbad.u-strasbg.fr/simbad/

${ }^{2}$ http: //leda.univ-lyonl.fr/

${ }^{3}$ https://ned.ipac.caltech.edu/
} 


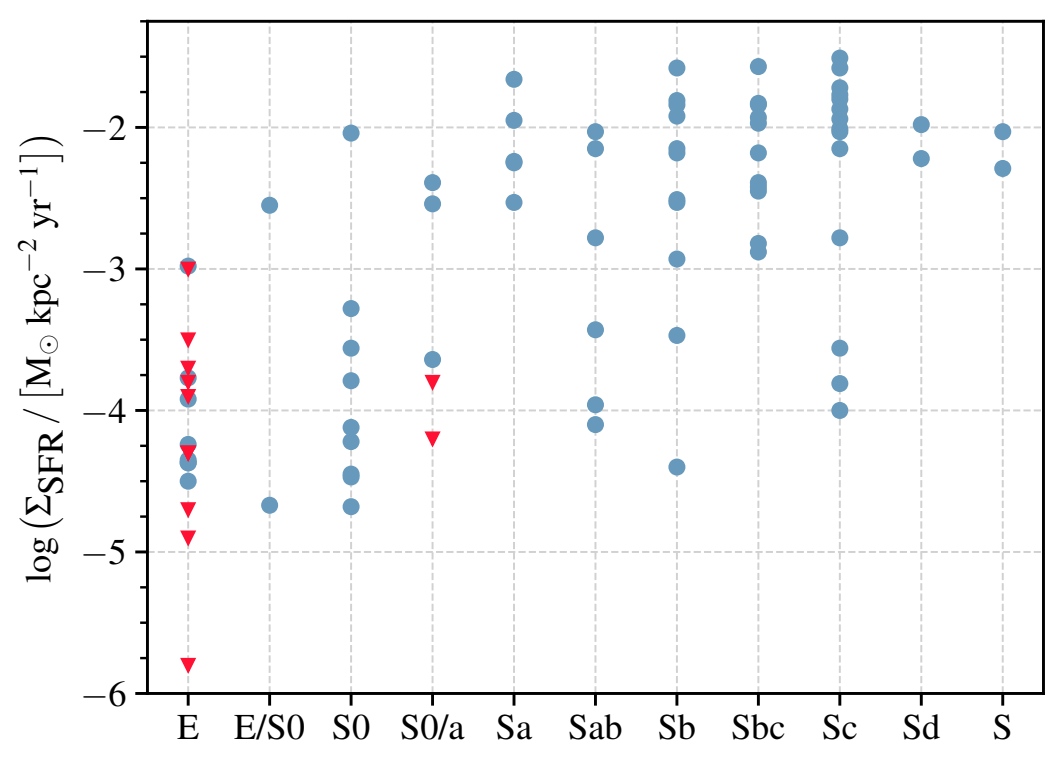

Figure 2: Local star formation rate in $1 \mathrm{kpc}$ region around supernovae vs. morphological type of the hosts for the SN sample from [16]. The upper limits on the local SFR are marked by red triangles.

- There is no statistically significant trend for the colour parameter.

- Trend in the stretch nuisance parameter $\alpha$ exists. Its value decreases from $\mathrm{E} / \mathrm{L}$ to $\mathrm{LS}$ galaxies (see Fig. 3).

- In old stellar population and with low dust environment, supernovae are intrinsically fainter after stretch and colour corrections. This conclusion contradicts the results obtained by [20] (see also [21] and references in it). However, our results are consistent within $1-\sigma$ for all subsamples, that makes them insignificant.

- Host galaxy morphology affects the residual dispersion in distance modulus on the Hubble diagram. It was noticed that SNe Ia in LS are more homogeneous with $\Delta \mu R M S=0.115 \pm$ 0.010 against $\Delta \mu R M S=0.138 \pm 0.007$ for the full sample.

\subsection{Pantheon supernova sample}

Cosmological supernova sample Pantheon consists of 1048 objects with redshifts up to $z \simeq$ 2.3 [3]. Pantheon also represents a compilation from several surveys: 177 nearby supernovae $(0.01<z<0.1), 335$ SDSS supernovae, 236 SNLS supernovae, 279 objects from Pan-STARRS survey and 21 HST supernovae. Pantheon is the largest cosmological SN sample to date. The main advantages of Pantheon compared to previous compilations are: an intercalibration between different surveys and a thorough investigation of systematic uncertainties.

To analyse how the morphological type of host galaxies affects the supernova luminosity and standardization parameters we, first, determined the host morphology according to the Hubble 


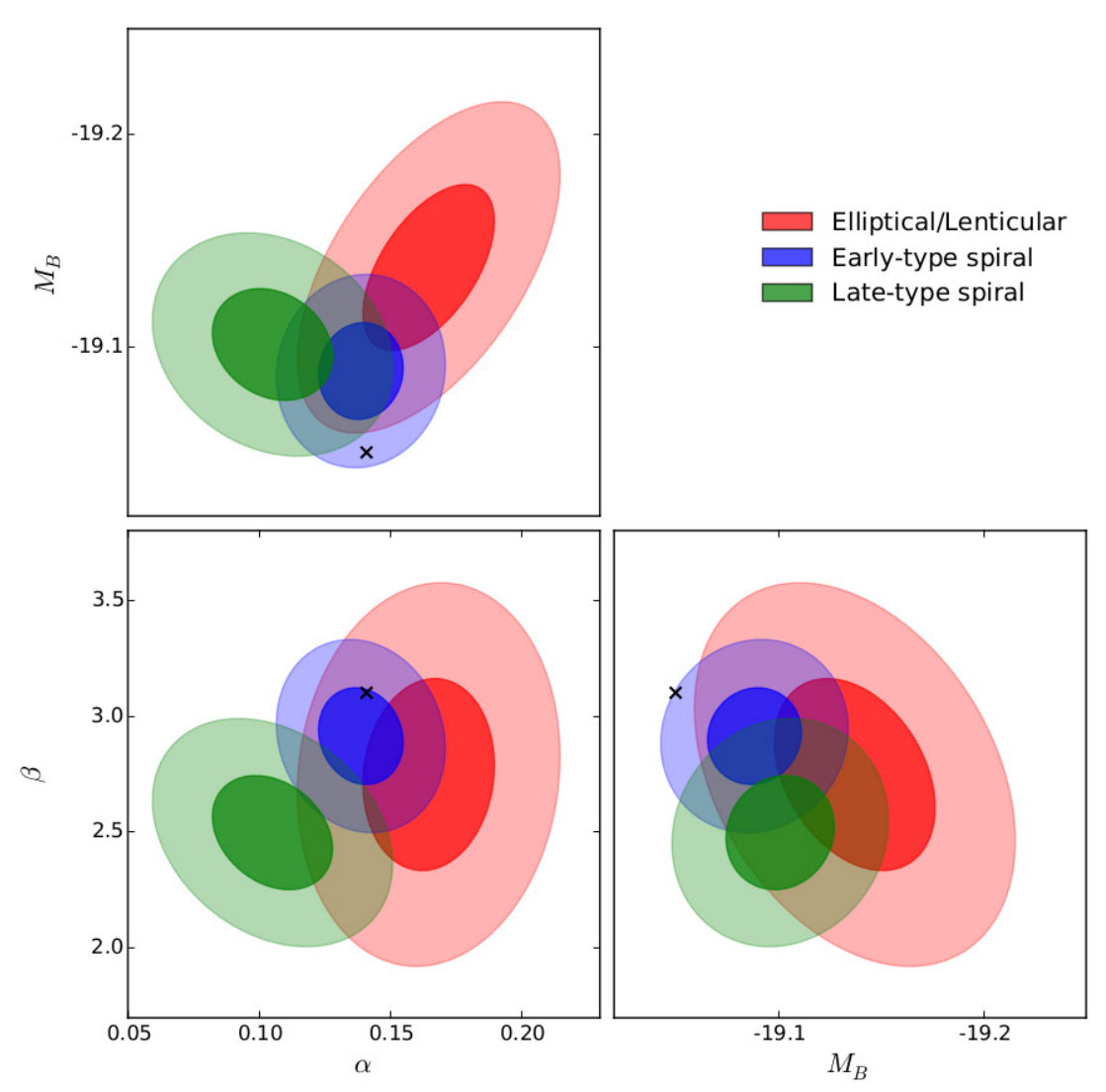

Figure 3: Joint confidence contours (1- and 2- $\sigma$ ) in two-parameter plots of $\alpha, \beta$, and $M_{B}$ for the Hubble diagram fits with Eq. 2.1 and fixed $\Lambda \mathrm{CDM}$ cosmology with $\Omega_{\Lambda}=0.705$, where SNe are split according to the host galaxy morphology into three categories (elliptical/lenticular, early-type spiral, and late-type spiral galaxies). The black crosses represent the best fitting values $\left(\alpha=0.141, \beta=3.101, M_{B}=-19.05\right)$ from [18].

classification. To do that, we used several astronomical databases (SIMBAD, HyperLEDA, NED) as well as individual publications.

Based on these sources we found the host morphology of 248 SNe Ia from the Pantheon sample. In cases when the morphological classification given by different sources is controversial, we made a visual classification. The final distribution of SN Ia hosts by morphological types is given in Fig. 4. As it is seen from the plot, the distribution is uneven. That is why we combined the "close" morphological types with each other (see Table 1). We considered four groups:

- E-S0/a - elliptical and lenticular galaxies (low star formation rate, old stellar population, low amounts of dust);

- Sa-Sb - early type spirals (tightly wound arms); 
Table 1: Distribution of host galaxies of Pantheon SNe Ia according to their morphological type.

\begin{tabular}{|c|c|c|c|c|c|c|c|c|c|c|c|c|}
\hline $\begin{array}{c}\text { E } \\
(20)\end{array}$ & $\begin{array}{c}\text { S0/E } \\
(17)\end{array}$ & $\begin{array}{c}\text { S0 } \\
(25)\end{array}$ & $\begin{array}{c}\text { S0/a } \\
(10)\end{array}$ & $\begin{array}{c}\text { Sa } \\
(25)\end{array}$ & $\begin{array}{c}\text { Sab } \\
(6)\end{array}$ & $\begin{array}{c}\text { Sb } \\
(45)\end{array}$ & $\begin{array}{c}\text { Sbc } \\
(12)\end{array}$ & $\begin{array}{c}\text { Sc } \\
(48)\end{array}$ & $\begin{array}{c}\text { Scd } \\
(1)\end{array}$ & $\begin{array}{c}\text { Sd } \\
(4)\end{array}$ & $\begin{array}{c}\text { S } \\
(6)\end{array}$ & $\begin{array}{c}\text { Irr } \\
(29)\end{array}$ \\
\hline
\end{tabular}

- Sbc-Sd — late type spirals (loosely wound and weak arms);

- Irr — irregular (no arms, no bulge, irregular profile, low surface brightness).

\subsection{1 $x_{1}$ and $c$ parameters}

The distribution of Pantheon supernova by $x_{1}$ parameter for four morphological groups is shown in Fig. 5a. It is seen that supernovae with low-stretch parameter prevail in elliptical and lenticular galaxies, middle stretch values are typical for spirals and highest $x_{1}$ we found for SNe Ia exploded in irregular galaxies. The same analysis but for $c$ parameter (Fig. 5b) did not reveal any dependencies. This is consistent with the previous results obtained on other SNe Ia samples (e.g., [21]).

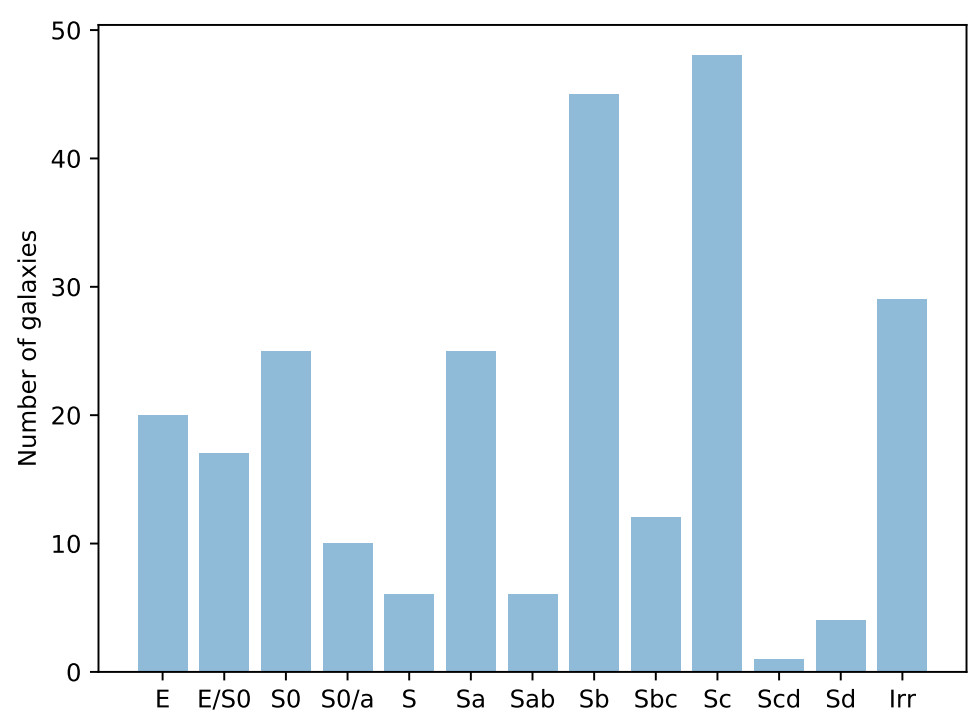

Figure 4: Histogram shows the distribution of host galaxies of Pantheon SNe Ia by morphological type according to the Hubble classification.

\subsubsection{Hubble diagram}

Using 248 SNe Ia from Pantheon, we plotted the Hubble diagram (Fig. 6) and calculated the residual dispersion in distance modulus for four groups (see Table 2). The standardization parameters and $\Omega_{\Lambda}$ are fixed at this step to the best-fit values in Pantheon analysis [3]. 

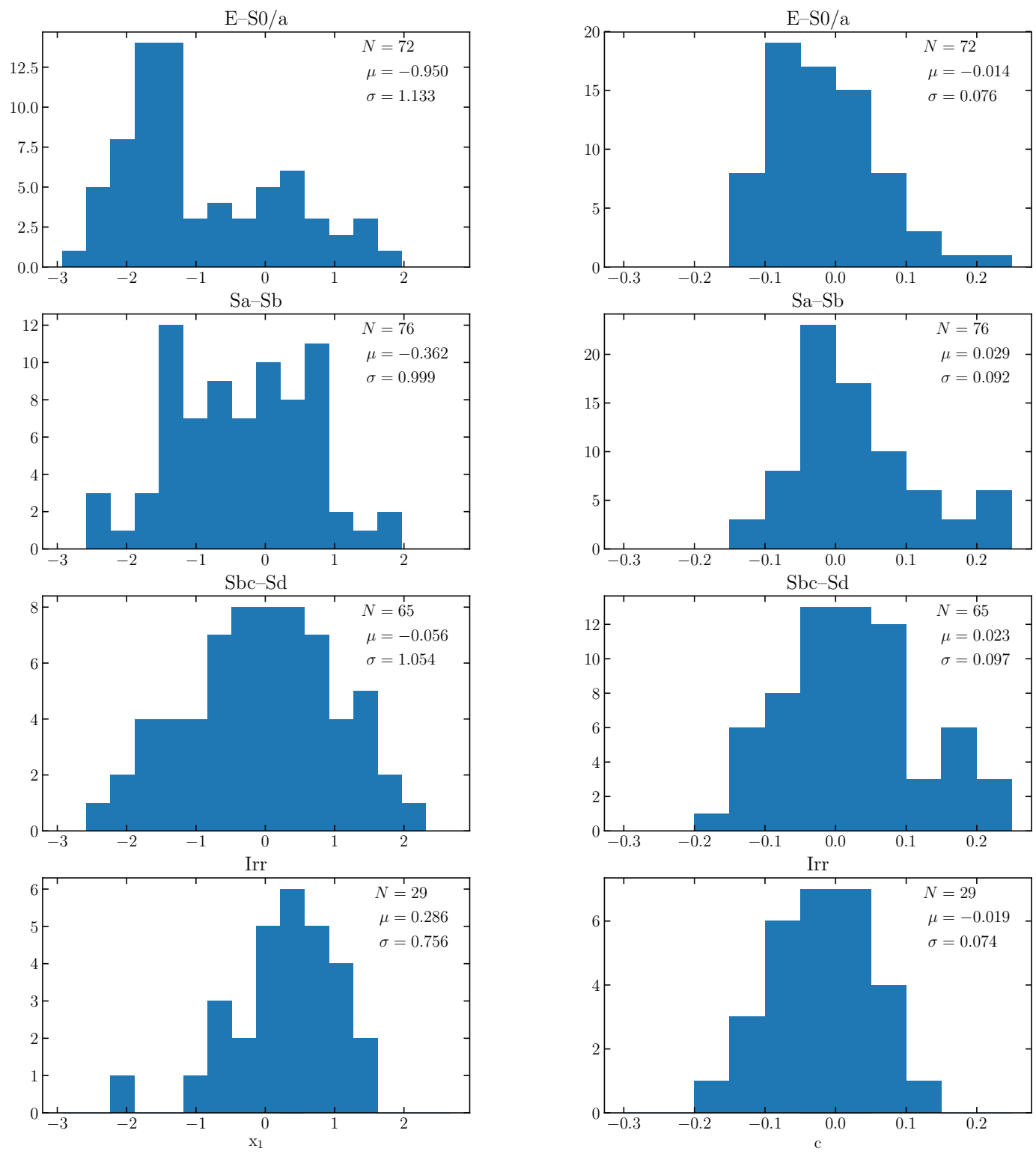

(a)

(b)

Figure 5: Distribution of $x_{1}$ (a) and $c$ (b) parameters for four morphological groups.

Let us define the difference between theoretical $\left(\mu_{i}^{t h}\right)$ and observed $\left(\mu_{i}\right)$ distance modulus for i-supernova as:

$$
\begin{gathered}
\Delta \mu_{i}=\mu_{i}^{t h}-\mu_{i} \\
\mu^{t h}=5 \log _{10} d_{\mathrm{L}}-5, \quad d_{\mathrm{L}}=\frac{c}{H_{0}}(1+z) \int_{0}^{z} \frac{d z^{\prime}}{\sqrt{\Omega_{\Lambda}+\Omega_{m}\left(1+z^{\prime}\right)^{3}}} .
\end{gathered}
$$

Then, for each group we estimate $w R M S$ [22]: 


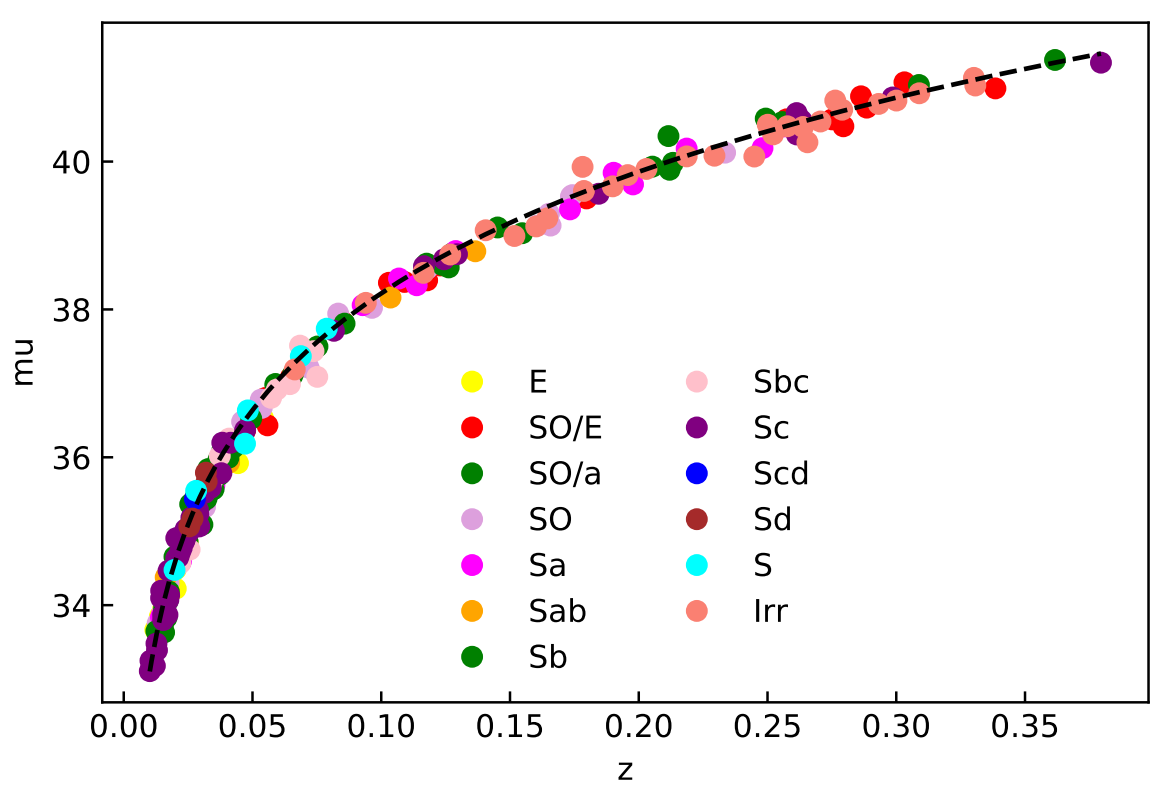

Figure 6: Hubble diagram for 248 Pantheon supernovae.

Table 2: $w R M S$ for four groups and in total for 248 Pantheon supernovae.

\begin{tabular}{|l|l|}
\hline Group & $\Delta \mu w R M S$ \\
\hline E-S0/a & 0.1484 \\
Sa-Sb & 0.1526 \\
Sbc-Sd & 0.1608 \\
Irr & 0.1358 \\
\hline Full sample & 0.1449 \\
\hline
\end{tabular}

$$
w R M S=\sqrt{\left(\sum_{i=1}^{N} w_{i}\right)^{-1} \sum_{i=1}^{N} w_{i} \Delta \mu_{i}^{2}},
$$

$$
w_{i}=\sigma_{i}^{-2}
$$

where $w_{i}$ is weight of i-supernova.

From Table 2 we could see that the residual dispersion in distance modulus differs for the supernovae exploded in different type of galaxies. So, $w R M S$ is smallest for $\mathrm{SNe}$ Ia in irregular galaxies. This could reflect the fact that $\mathrm{SN}$ properties depends on host morphology — in some 
hosts supernovae are more "standard" and therefore their dispersion on the Hubble diagram is smaller.

\section{Conclusions}

We studied the influence of host galaxy morphology on light curve parameters of the JLA and Pantheon Type Ia supernovae. We confirmed that the stretch parameter of SNe Ia is correlated with the host galaxy type. The supernovae with lower stretch are hosted mainly by elliptical and lenticular galaxies. The correlation for colour parameter has not been found. This conclusion accords to other researches that characterise the environment with the local parameters like local star formation rate, local colour and etc.

The host galaxy morphology affects the residual dispersion on the Hubble diagram. JLA SNe Ia in late-type spiral galaxies are more homogeneous in comparison with others. Therefore, we confirm that the supernova properties depend on their environment and the incorporation of a host galaxy term into the Hubble diagram fit is expected to be crucial for future cosmological analyses.

\section{Acknowledgements}

M. Pruzhinskaya acknowledges support from RSF grant 18-72-00159 for analysis of the environmental effects for Pantheon supernova sample. A. Novinskaya acknowledges support from the Program of Development of M.V. Lomonosov Moscow State University (Leading Scientific School "Physics of stars, relativistic objects and galaxies").

\section{References}

[1] A. G. Riess, A. V. Filippenko, P. Challis, A. Clocchiatti, A. Diercks, P. M. Garnavich et al., Observational Evidence from Supernovae for an Accelerating Universe and a Cosmological Constant, AJ 116 (Sept., 1998) 1009-1038.

[2] S. Perlmutter, G. Aldering, G. Goldhaber, R. A. Knop, P. Nugent, P. G. Castro et al., Measurements of Omega and Lambda from 42 High-Redshift Supernovae, ApJ 517 (June, 1999) 565-586.

[3] D. M. Scolnic, D. O. Jones, A. Rest, Y. C. Pan, R. Chornock, R. J. Foley et al., The Complete Light-curve Sample of Spectroscopically Confirmed SNe Ia from Pan-STARRS1 and Cosmological Constraints from the Combined Pantheon Sample, ApJ 859 (June, 2018) 101, [1710. 00845 ].

[4] M. V. Pruzhinskaya and S. M. Lisakov, How supernovae became the basis of observational cosmology, Journal of Astronomical History and Heritage 19 (Dec, 2016) 203-215, [1 608.04192 ].

[5] B. W. Rust, Use of supernovae light curves for testing the expansion hypothesis and other cosmological relations, Ph.D. thesis, Oak Ridge National Lab., TN., 1974.

[6] I. P. Pskovskii, Light curves, color curves, and expansion velocity of type I supernovae as functions of the rate of brightness decline, Soviet Ast. 21 (Dec., 1977) 675-682.

[7] Y. P. Pskovskii, Photometric classification and basic parameters of type I supernovae, Soviet Ast. 28 (Dec., 1984) 658-664.

[8] M. M. Phillips, The absolute magnitudes of Type IA supernovae, ApJL 413 (Aug., 1993) L105-L108. 
[9] M. Hamuy, M. M. Phillips, N. B. Suntzeff, R. A. Schommer, J. Maza and R. Aviles, The Absolute Luminosities of the Calan/Tololo Type IA Supernovae, AJ 112 (Dec., 1996) 2391, [astro-ph/9609059].

[10] R. Tripp, A two-parameter luminosity correction for Type IA supernovae, A\&A 331 (Mar., 1998) $815-820$.

[11] J. Guy, P. Astier, S. Baumont, D. Hardin, R. Pain, N. Regnault et al., SALT2: using distant supernovae to improve the use of type Ia supernovae as distance indicators, A\&A 466 (Apr., 2007) 11-21.

[12] G. Aldering, G. Adam, P. Antilogus, P. Astier, R. Bacon, S. Bongard et al., Overview of the Nearby Supernova Factory, in SPIE Conference Series, vol. 4836, pp. 61-72, Dec., 2002.

[13] M. Rigault, V. Brinnel, G. Aldering, P. Antilogus, C. Aragon, S. Bailey et al., Strong Dependence of Type Ia Supernova Standardization on the Local Specific Star Formation Rate, arXiv e-prints (Jun, 2018) arXiv:1806.03849, [1806.03849].

[14] J. Meyers, G. Aldering, K. Barbary, L. F. Barrientos, M. Brodwin, K. S. Dawson et al., THEHUBBLE SPACE TELESCOPECLUSTER SUPERNOVA SURVEY. III. CORRELATED PROPERTIES OF TYPE ia SUPERNOVAE AND THEIR HOSTS AT 0.9 less z less 1.46, The Astrophysical Journal 750 (apr, 2012) 1.

[15] H. Domínguez Sánchez, M. Huertas-Company, M. Bernardi, D. Tuccillo and J. L. Fischer, Improving galaxy morphologies for SDSS with Deep Learning, Monthly Notices of the Royal Astronomical Society 476 (02, 2018) 3661-3676, [http://oup.prod.sis.lan/mnras/article-pdf/476/3/3661/24507962/sty338.pdf].

[16] M. Rigault, G. Aldering, M. Kowalski, Y. Copin, P. Antilogus, C. Aragon et al., Confirmation of a Star Formation Bias in Type Ia Supernova Distances and its Effect on the Measurement of the Hubble Constant, ApJ 802 (Mar, 2015) 20, [1412.6501].

[17] M. Roman, D. Hardin, M. Betoule, P. Astier, C. Balland, R. S. Ellis et al., Dependence of Type Ia supernova luminosities on their local environment, Astronomy and Astrophysics 615 (Jul, 2018) A68, [1706.07697].

[18] M. Betoule, R. Kessler, J. Guy, J. Mosher, D. Hardin, R. Biswas et al., Improved cosmological constraints from a joint analysis of the sdss-ii and snls supernova samples", A\&A 568 (2014) 32.

[19] V. Henne, M. V. Pruzhinskaya, P. Rosnet, P.-F. Léget, E. E. O. Ishida, A. Ciulli et al., The influence of host galaxy morphology on the properties of Type Ia supernovae from the JLA compilation, New A 51 (Feb., 2017) 43-50, [1608.03674].

[20] M. Hicken, W. M. Wood-Vasey, S. Blondin, P. Challis, S. Jha, P. L. Kelly et al., Improved Dark Energy Constraints from 100 New CfA Supernova Type Ia Light Curves, ApJ 700 (Aug., 2009) 1097-1140, [0901.4804].

[21] M. Sullivan, A. Conley, D. A. Howell, J. D. Neill, P. Astier, C. Balland et al., The dependence of Type Ia Supernovae luminosities on their host galaxies, MNRAS 406 (Aug., 2010) 782-802, [1003.5119].

[22] S. Blondin, K. S. Mandel and R. P. Kirshner, Do spectra improve distance measurements of Type Ia supernovae?, A\&A $\mathbf{5 2 6}$ (Feb, 2011) A81, [1 012 . 0005 ]. 


\section{DISCUSSION}

WOLFGANG KUNDT's Comment: Maria, you mentioned a thermally exploding white dwarf that gave rise to a SN of type Ia. There is the problem that for non-negligible gravity, explosions driven by non-relativistic pistons cool too fast for escape motion, i.e recollapse before expansion, like fountains. You should allow your white dwarf to tap a relativistic reservoir.

JIM BEALL: Do you find evidence of the possible different constitution of the SN depending on WD-WD or opposite to WD-normal stars?

MARIA PRUZHINSKAYA: By now we do not know which mechanism (SD or DD) works or if both in which proportion. From the observational point of view, polarimetric and spectropolarimetric observations could shed light on this problem. In case of DD explosion, the specific angular momentum of matter is higher than in the SD scenario. This could lead to an anisotropy of the explosion and asymmetrical loss of envelope and, consequently, a significant polarization of optical radiation is expected. Thereby, the registration of significant (more than 2\%) polarization can be an independent argument for a model of merging white dwarfs. It should be noted that the continuum polarization depends on the geometry of the explosion but line polarization associates with distribution of matter around supernovae. 